\title{
Interferometric time delay correction for Fourier transform spectroscopy in the extreme ultraviolet
}

\author{
Yijian Meng, Chunmei Zhang, Claude Marceau, A. Yu. Naumov, P. B. Corkum and D. M. Villeneuve \\ Joint Attosecond Science Laboratory, National Research Council and University of Ottawa, Ottawa, Canada
}

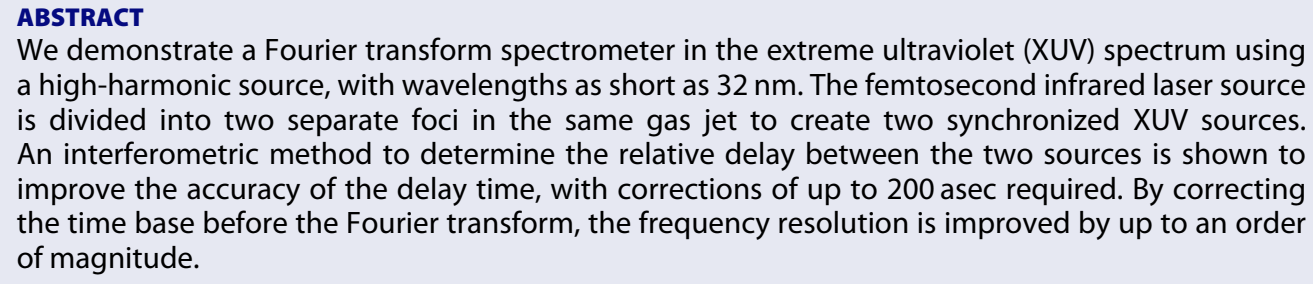

ARTICLE HISTORY

Received 9 December 2015

Accepted 4 March 2016

\section{KEYWORDS}

Fourier transform

spectroscopy; time delay

correction; XUV; high-order

harmonics

\section{Introduction}

Fourier transform spectroscopy (FTS) is a powerful tool for measuring the absorption spectrum of chemical compounds (1), particularly in spectral regions where focal plane array detectors are not available. In FTS, the light source is interferometrically divided into two beams that interfere on the detector as a function of relative time delay. The resulting signal is Fourier transformed from time to frequency to obtain the power spectrum of the source. The practical spectral resolution of FTS is determined by the total range of time retardation and the uniformity of the delay steps. Traditionally, FTS was done in the infrared/UV region, where a delay step and stability of $>100 \mathrm{~nm}$ is sufficient. In recent years, FTS has been applied to shorter wavelength sources $(2,3)$. In the extreme ultraviolet (XUV) spectral range, FTS can be used to study molecular dissociation (4). And at even shorter wavelengths in the X-ray water window, which covers the spectral range between the carbon K-edge $(4.4 \mathrm{~nm})$ and oxygen K-edge $(2.34 \mathrm{~nm})$, chemical bond information can be extracted from the absorption spectrum $(5,6)$.

A promising coherent light source in the XUV and $\mathrm{X}$-ray range is high harmonic generation (HHG). In comparison to sources such as synchrotrons or free electron lasers, HHG sources are much more accessible as they can fit on a single optical table. Because of the lack of broadband beamsplitters in the XUV range, two different approaches are taken to create replicas of the XUV radiation for FTS. (1) The wavefront can be spatially split in vacuum after HHG using split mirrors $(4,7,8)$. (2) The infrared laser beam can be split before HHG using optical beamsplitters, creating two spatially separated HHG sources in the gas jet $(2,3,9,10)$. The latter approach has the advantage that temporal delay of the replicas is performed at the infrared laser wavelength, not the XUV. Unlike a Michelson interferometer, in both of these approaches, the replicas are not collinear. This leads to interference fringes when the two replicas overlap at the detector. The detector must be able to resolve the fringes spatially in order to record an FTS signal.

However, using FTS at shorter wavelengths has highlighted the technical challenges (2). An accurate measurement of the spectrum requires that the delay steps must be accurate to much less than a wavelength. For XUV or $\mathrm{X}$-ray sources, this demands that the delay be accurate to the nanometer scale. This requirement is very demanding with any interferometric set-up due to mechanical vibrations, air fluctuations and non-uniform translation stages. We illustrate this problem with a simple numerical simulation. In Figure 1, we show the effect of a nonuniform delay on the quality of the retrieved power spectrum using a HHG source. A realistic attosecond pulse train is calculated using the strong field approximation $(11,12)$. We create a non-uniform delay by adding Gaussian noise to the uniform delay step. When the autocorrelation function is Fourier transformed, the resulting power spectrum becomes distorted. As the delay time noise increases to $20 \mathrm{asec}$, standard deviation, spectral peaks near $40 \mathrm{eV}$ are no longer distinguishable from the

CONTACT D. M. Villeneuve David.Villeneuve@uottawa.ca

(c) Copyright of the Crown in Canada 2016 National Research Council 


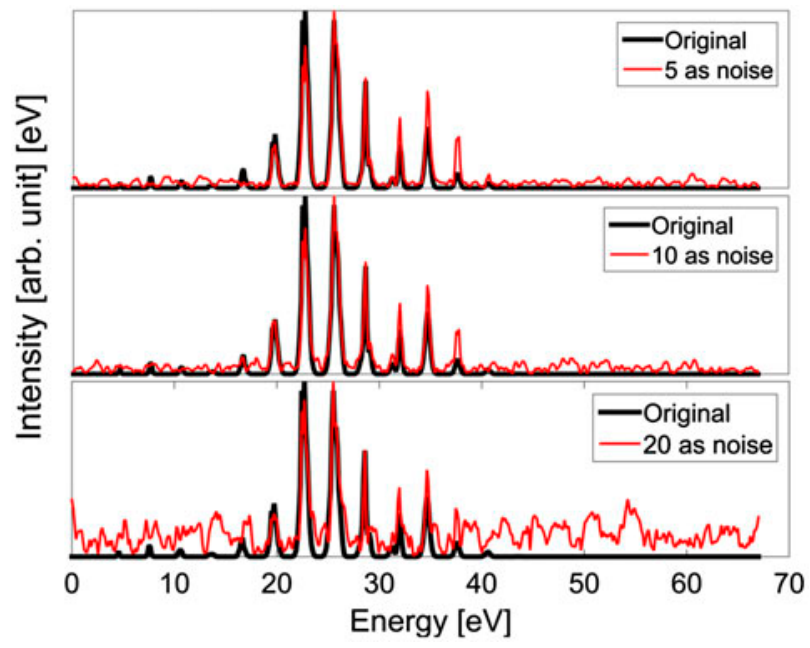

Figure 1. Simulated power spectrum determined by Fourier transform spectrometry. An attosecond pulse train in the time domain is calculated using a strong field approximation model. Autocorrelations are then calculated to yield the power spectra. The time base of delayed beam is then statistically jittered to simulate jitter in the experimental interferometer. The black line is the original power spectrum from the autocorrelation at uniform time delay. The red line uses the same mathematical procedure as the black line but uses data at non-uniform time delay. The deviation from uniform delay follows a Gaussian distribution. The standard deviations are 5, 10 and 20 asec in the top, middle and bottom figures, respectively.

background noise. We conclude from this example that the time delay accuracy must be better than 10 asec, or $3 \mathrm{~nm}$ in length, in order to spectrally resolve a peak at $40 \mathrm{eV}$.

The existing solutions to this demand for high accuracy delays have been to mechanically improve the optical set-up, for example, vibration isolation, high precision delay stages and operating the optical components in vacuum. However, it becomes increasingly difficult to isolate vibrations for delays of only a few $\mathrm{nm}$. Similar to the Brault algorithm used in FTS in the UV range (13), we develop an interferometric method to improve the quality of FTS in the XUV or X-ray range, by recording the actual time delay at each delay step, and resampling the time base to a uniform spacing.

\section{Method}

The experimental set-up, shown in Figure 2, is based on one used to perform hyperspectral coherent diffractive imaging in the XUV (14). The XUV radiation is produced by HHG using $5 \mathrm{~mJ}$ from a Ti:Sapphire laser at a $100 \mathrm{~Hz}$ repetition rate with a pulse duration of $50 \mathrm{fs}$. To create the time delay between the two identical laser pulses, we use a pair of Wollaston prisms to create an almostcollinear delay set-up at the $800 \mathrm{~nm}$ laser wavelength. Each Wollaston prism consists of two $5^{\circ}$ calcite wedges with perpendicular optical axis. The first half-wave plate rotates the polarization to $45^{\circ}$ from the vertical direction. The first Wollaston prism separates the vertical and horizontal polarization components of the incoming beam. By translating the Wollaston prism perpendicularly to the beam using a linear translation stage (Newport MFAPPD), we simultaneously increase and decrease the group delay along the two optical axes. This creates retardation between the two beams of perpendicular polarizations. The second Wollaston prism compensates for the delay difference and the relative angle between the two beams. In addition, by rotating the second Wollaston prism, we can fine tune the relative angle between the two beams. Using a second half-wave plate, we rotate the beam polarization back to $\pm 45^{\circ}$ from the vertical direction. The two beams then go through a linear polarizer at Brewster's angle to make the polarization of both beams parallel. The reflected component from the polarizer is focused into a pulsed gas jet of argon with a $250 \mathrm{~mm}$ focal length lens. By adjusting the relative angle between the two beams, we produce two foci of $30 \mu \mathrm{m}$ diameter that are horizontally separated by $180 \mu \mathrm{m}$. The two foci are placed on a line that is perpendicular to the gas jet expansion direction, to ensure that both foci experience the same gas density. The two HHG sources diverge and overlap in the far field, creating an interference pattern.

There is a trade-off in choosing the separation between the two laser foci in the gas jet. As the separation between the foci increases, the fringe separation in the far-field interference pattern gets smaller, and eventually cannot be spatially resolved. As the separation decreases, the $800-$ $\mathrm{nm}$ laser fields begin to overlap spatially in the gas jet. The HHG process is very sensitive to perturbing fields, even with less than $1 \%$ intensity (15). As the fields begin to overlap in the foci, a cross-modulation appears between the two HHG sources (2). The choice of $180 \mu \mathrm{m}$ separation achieves a balance between these two objectives.

We image the far-field pattern using a combination of microchannel plate detector (MCP) and high dynamic range camera (PCO 1600). For each delay position, we integrate 10 frames over a 2 -second exposure time. We estimate the illumination at each delay step to be in the order of $10^{11}$ photons. For some of the measurements, an XUV spectrometer was used to disperse the spectrum onto the detector.

Because the two HHG generating regions are spatially separated in the vertical direction, an interference pattern is also observed at the detector in the vertical direction. For a detector at distance $L$ from the HHG sources with separation $D$ and whose wavelength is $\lambda$, the detector spatial resolution $r$ must be $r \ll L \lambda /(2 \pi D)$. As the relative delay between the two XUV replicas is varied, the fringe pattern moves vertically. At the same time, the 


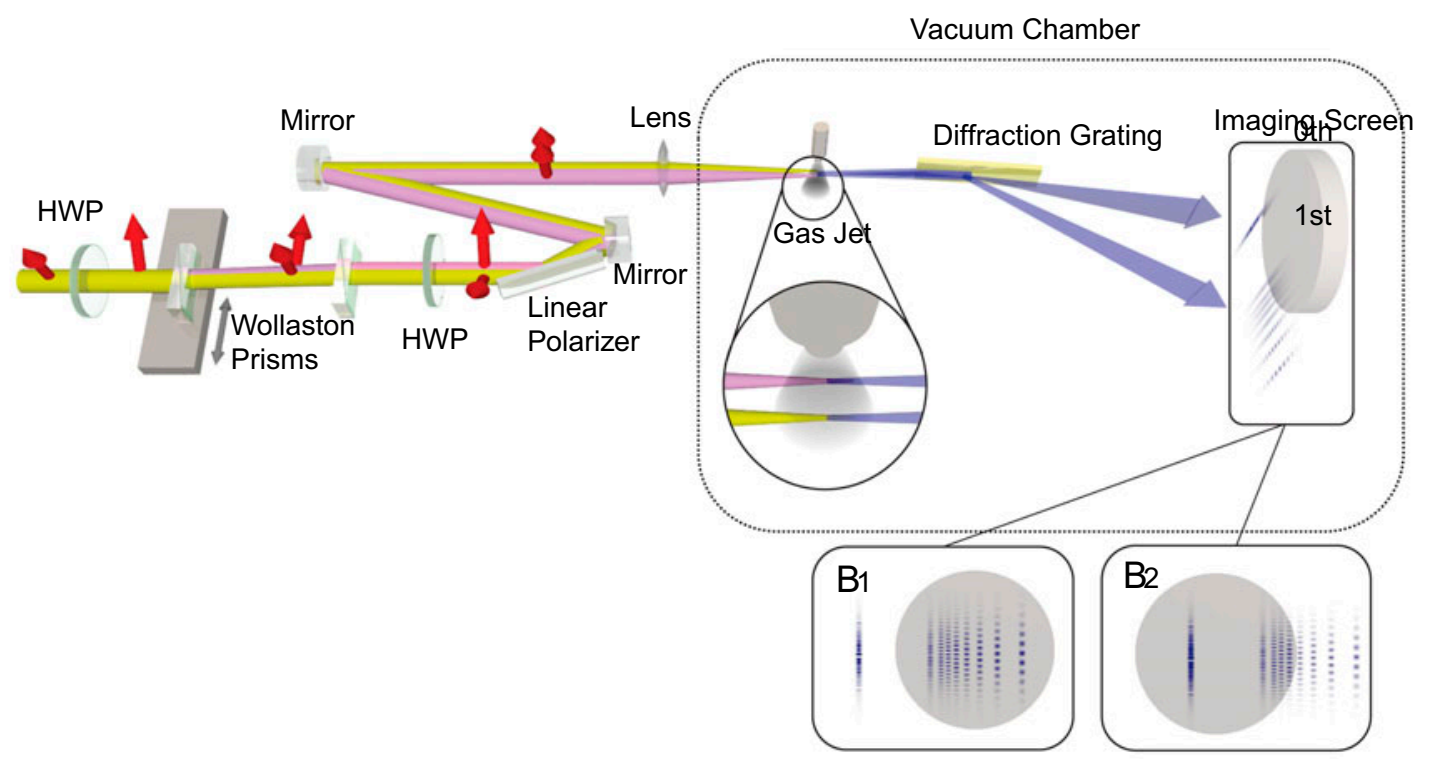

Figure 2. Part A shows the experimental set-up for FTS. The $800 \mathrm{~nm}$ laser beam is split and delayed by two Wollaston prisms. The two beams are then focused into a gas jet to produce two spatially separated high harmonic sources. Part $B_{1}$ presents the position of the imaging detector for recording the first-order diffraction, and part $B_{2}$ shows the arrangement used to simultaneously record the zero-order beam plus first-order diffraction for time delay correction.

intensity at any detector pixel goes up and down as the fringes pass over. The Fourier transform of the intensity vs time delay at each pixel gives the power spectrum of the XUV radiation at that pixel.

Here we demonstrate how to accurately record the relative delay between the two XUV replicas. An edge of the beam is reflected with a grazing-incidence metal mirror onto an XUV grating which disperses the HHG radiation into harmonic orders on the detector. The remainder of the beam can be used for imaging experiments. Alternatively, the entire XUV beam can be sent to the diffraction grating, and the zero-order reflection can be used for imaging experiments. In both cases, the diffracted harmonic orders show interference fringes in the vertical direction. See the insets in Figure 2.

By recording the intensity profile $I_{q}(y)$ of the interference fringes of harmonic order $q$ in the direction of the fringes $y$, we can measure the phase difference between the two beams. To extract the phase difference, we take a spatial Fourier transform of $I_{q}(y)$. The phase of the Fourier transform gives the position of the fringes and hence the phase delay modulo $2 \pi$ between the two XUV replicas. We have also fitted the fringes with a sine function, which gives similar result to the Fourier transform. We correct the phase jump by adding $2 \pi$ when the absolute jumps between the consecutive elements are greater than a tolerance level of $\pi$ radians.

During the experiment, the computer moves the Wollaston translation stage, attempting to set a relative time delay $t_{i}$. At each step $i$, the phase difference is measured as described above, and determines the actual corrected time delay $t_{i}^{c}$ for this step. This creates a one-to-one mapping between the computer-controlled non-uniform delay $t_{i}$ and the corrected time delay $t_{i}^{c}$. With an ideal experimental set-up, $t_{i}=t_{i}^{c}$, but in an actual experiment, the mapping will deviate from this simple relationship.

In an imaging experiment, we then interpolate the measured signal at each pixel onto a uniform time delay axis through linear interpolation. This effectively reduces the error due to non-uniform time delay steps. In addition, we can reduce the exposure time and measure multiple frames at one delay. This approach effectively reduces delay jitter at a single delay position. However, the photon flux of the signal drops linearly with the reduced exposure time. This increases the shot noise which could be detrimental to the measurement at the lower intensity region of the measured signal.

\section{Results and discussion}

\subsection{FTS of a monochromatic source}

First, we demonstrate the accuracy of this technique to correct the time delay between the XUV replicas. We use an XUV grating to diffract the HHG radiation into harmonic orders in the horizontal direction. The MCP then records the XUV spectrum in the horizontal direction and the fringes in the vertical direction. Figure 3(a) shows the first-order diffraction recorded at the detector. The dispersed wavelengths are spatially separated and 

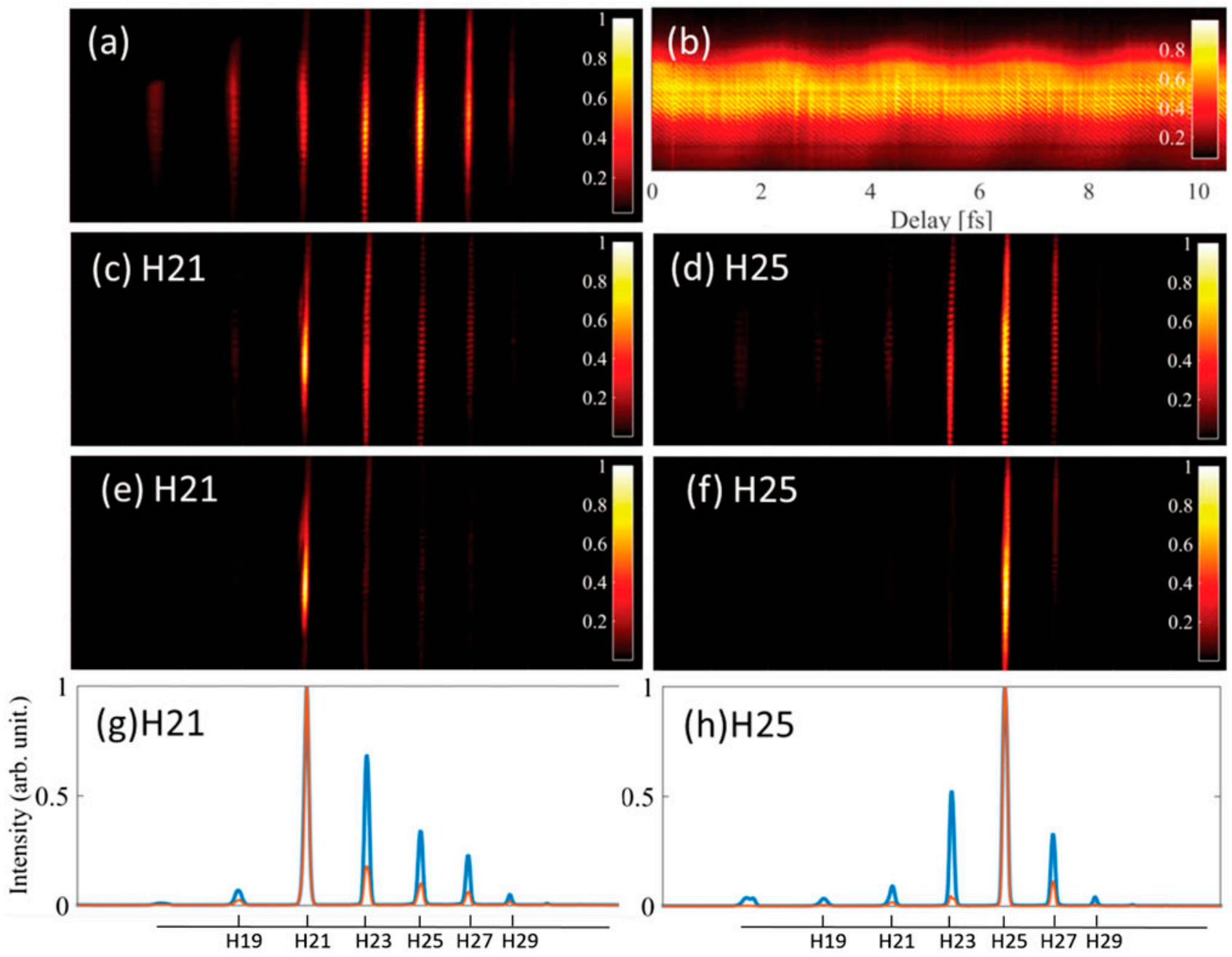

Figure 3. (a) Image from the 2D detector showing a spectrum that is dispersed horizontally by the diffraction grating. Each vertical line is a harmonic order from the HHG source. In the vertical direction, interference fringes can be seen, caused by interference between the two vertically displaced HHG sources. (b) A vertical lineout through $\mathrm{H} 21$ is plotted as a function of delay time. This scan is used to correct the time delay. (c) Detector image digitally filtered for $\mathrm{H} 21$, without time delay correction. (d) Detector image digitally filtered for $\mathrm{H} 25$, without time delay correction. (e-f) Digitally filtered images after time delay correction. $(\mathrm{g}-\mathrm{h}$ ) Vertically integrated intensity of frequency filtered image before (blue) and after (red) time delay correction.

become quasi-monochromatic at the imaging screen. We therefore should expect the Fourier transform at each pixel to produce a quasi-monochromatic spectrum.

We delay the two sources with a 6-asec step size over a total delay of $11 \mathrm{fs}$ using the Wollaston translation stage. The total delay range determines the spectral resolution of $0.4 \mathrm{eV}$. Due to experimental considerations, this time delay step size is not precise. We pick harmonic 21 as the time delay reference for all harmonic orders. Figure 3(b) shows the evolution of the vertical interference fringes at $\mathrm{H} 21$ as a function of delay $t_{i}$. Figure 4 shows the amount of correction $t_{i}^{c}-t_{i}$ vs. $t_{i}$. This reveals a nonlinearity which is partly due to the translation stage, particularly around $4 \mathrm{fs}$, and smaller fluctuations which could be due to atmospheric fluctuations or vibrations. Figure 4 shows the time correction derived from three different harmonic orders. All three measurements show the same trend. There is a slight order-dependent deviation that has a periodicity of $2.6 \mathrm{fs}$, the period of the $800 \mathrm{~nm}$ laser field. We attribute this periodicity to the influence of the weak shoulders of the two foci overlapping in the gas jet. It is known that such weak fields can modify the emission time of the harmonics; this technique is used for in situ attosecond pulse characterization techniques (15). Here, as one infrared pulse is delayed relative to the other, the weak field is delayed, leading to a periodic modulation of the emission time. The shift of the modulation in the horizontal direction between harmonic orders is a measure of the emission time of the harmonic orders (16). The periodic change in the emission time caused 


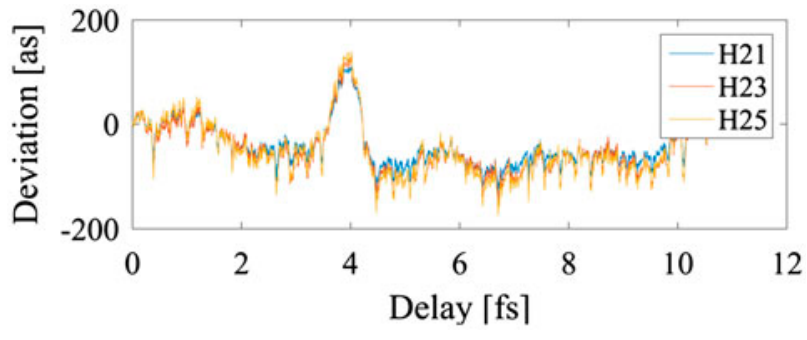

Figure 4. The amount of time correction that was determined by the measurement shown in Figure $3(\mathrm{~b})$. The vertical axis is $t_{i}-t_{i}^{c}$. Time corrections for three different harmonic orders are shown. The large deviation at $4 \mathrm{fs}$ is probably caused by nonlinearity of the translation stage that moved the Wollaston prism. The time delay error is dominated by non-uniform motion of the translation stage. The standard deviation of the jitter at a fixed delay position was 10 asec with a $15 \mathrm{~s}$ integration time, considerably smaller than the translation stage error.

by the slight overlap of the foci is probably the limiting factor in FTS using high harmonic emission.

We now show how the time delay correction can improve the spectrum extracted by FTS. The intensity at every pixel in the image shown in Figure 3(a) is recorded at each time delay $t_{i}$ to give $I_{x y}\left(t_{i}\right)$. The intensity at each pixel is then Fourier transformed to determine the spectrum at that pixel, i.e. $I_{x y}(\omega)=\mathrm{FT}_{t \rightarrow \omega}\left(I_{x y}(t)\right)$. For $t$, we first use the uncorrected $t_{i}$. The hyperspectral image $I_{x y}(\omega)$ is then digitally filtered to select only those frequencies at $\mathrm{H} 21$ (Figure 3(c)) and H25 (Figure 3(d)). It is evident that other harmonic orders are present in these spectra, due to the non-uniform time delay axis $t_{i}$.
We then interpolate $I_{x y}\left(t_{i}\right) \rightarrow I_{x y}\left(t_{i}^{c}\right)$ and again perform the Fourier transform to get the hyperspectral image and digitally filter for H21 (Figure 3(e)) and H25 (Figure $3(\mathrm{f})$ ). The contrast of the digitally selected harmonic orders is now improved. Figures $3(\mathrm{~g}-\mathrm{h})$ show lineouts of the spectra with time delay corrections applied, showing that a contrast of 5 is achieved for $\mathrm{H} 21$, and a contrast of 10 for $\mathrm{H} 25$.

\subsection{FTS of a broadband source}

In the previous section, we relied on a diffraction grating to separate the harmonic orders from the HHG sources to demonstrate the improvement given by the time delay correction procedure. We now mimic an actual hyperspectral imaging experiment in which all harmonic orders are present at every pixel. To do this at the same time as recording the dispersed harmonics for the time delay correction, we used the zero-order diffraction order from the grating. Figure 5(a) shows the zero-order reflection on the imaging detector. It is elongated vertically because the spherical diffraction grating focuses more in the horizontal direction. We again select $\mathrm{H} 21$ from the firstorder diffraction (not shown) to provide the time delay correction. The two HHG sources are delayed with a 30 -asec step size over a total delay of $37 \mathrm{fs}$, giving a spectral resolution of $0.11 \mathrm{eV}$. Figure $5(\mathrm{~b})$ shows the evolution of a vertical slice of the zeroth order as the time delay between the HHG sources changes. The diagonal stripes are due to the nature of the HHG emission as a
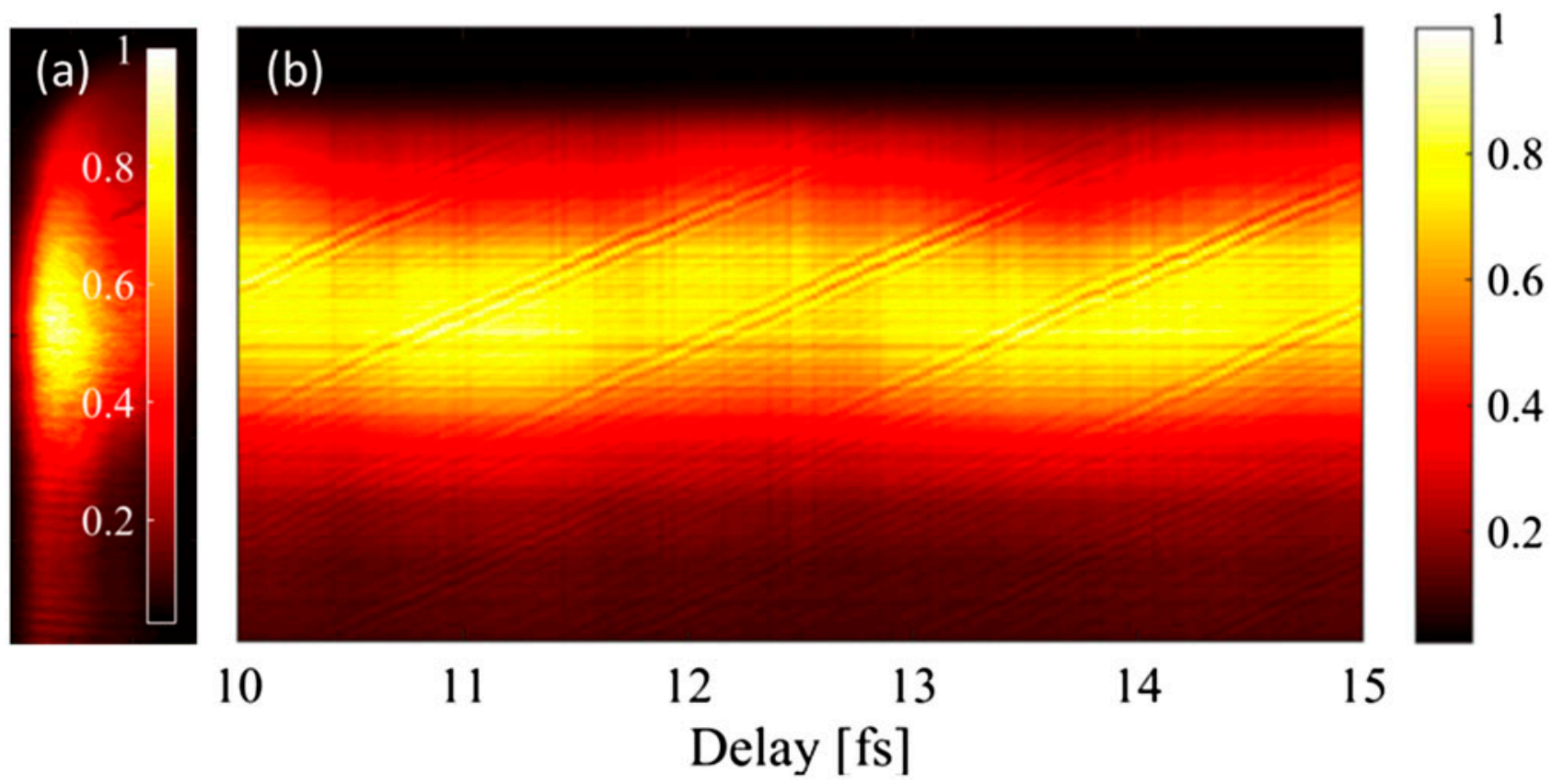

Figure 5. (a) Raw image of the zero-order reflection from the diffraction grating. The beam appears elongated because the spherical diffraction grating focuses more in the horizontal direction. (b) Time evolution of a vertical slice from (a). The diagonal stripes show the train of attosecond pulses created by the HHG sources. 


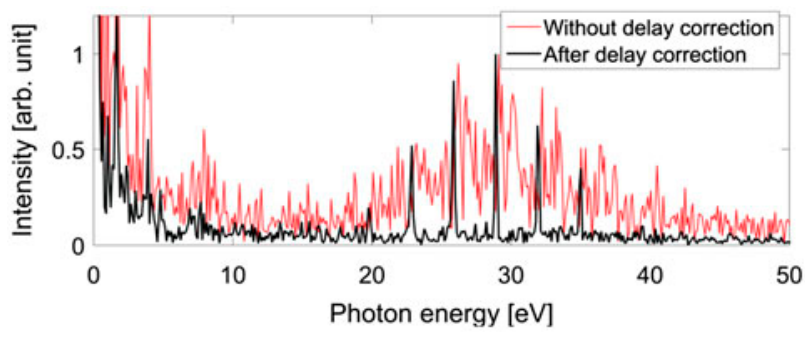

Figure 6. Normalized power spectrum at a single camera pixel from the zero-order image. The red line shows the power spectrum retrieved using the non-uniform time delays $t_{i}$. The black line shows the power spectrum after applying the time delay correction $t_{i}^{c}$.

train of attosecond pulses separated by half of the optical period of the $800 \mathrm{~nm}$ laser field, or about $1.3 \mathrm{fs}$. This is effectively an autocorrelation of the attosecond pulse train. The stripes are tilted because each vertical position experiences a different time delay between the HHG sources. Unlike the previous section in which the harmonic orders were dispersed with a grating, no fringes are evident in the vertical direction, due to the polychromatic nature of the HHG emission.

Again, we take the Fourier transform of the intensity vs. time delay at each pixel, $I_{x y}(\omega)=\mathrm{FT}_{t \rightarrow \omega}\left(I_{x y}(t)\right)$, to determine the XUV spectrum incident on each pixel. Figure 6 shows the power spectrum that is retrieved with and without time delay correction. Before correction, the power spectrum is noisy and has no distinct harmonic peaks. The power spectrum after correction shows wellseparated and evenly spaced harmonic peaks. This spectrum matches the results recorded with the grating spectrometer, where discrete harmonic peaks are observed. The shortest wavelength we can resolve is $32 \mathrm{~nm}$, corresponding to $\mathrm{H} 25$. The width of the high harmonic orders is about $0.33 \mathrm{eV}$ using FTS (Figure 6) compared with $0.40 \mathrm{eV}$ using the grating spectrometer (Figure $3(\mathrm{a})$ ). The fact that the spectral resolution using FTS is somewhat better than that using the grating spectrometer is probably due to the slit width of the grating spectrometer.

\section{Conclusion}

We have demonstrated a Fourier transform spectrometer operating in the XUV, with wavelengths as short as $32 \mathrm{~nm}$, based on high-harmonic sources. By interferometrically measuring the actual time delay between the two XUV sources, we were able to improve the spectral selectivity by about a factor of five. This technique can be extended to HHG sources with higher photon energies, permitting FTS and hyperspectral imaging in the $\mathrm{X}$-ray water window. The ultimate limitation of applying two high-harmonic sources to shorter wavelengths is the phase modulation of the XUV emission due to the weak overlap of the two infrared foci. To reduce this problem, adaptive optics will be needed to produce two independent laser foci.

In the present experiment, we used only a single harmonic order to correct the time delay. This limits the timing jitter at any delay position to give a phase shift of less than $2 \pi$. Because the phase shift is proportional to the frequency, $\phi_{q} \propto q \omega_{0} t$, this gives a set of equations which can determine $t$ over a phase shift range greater than $2 \pi$. This would permit a larger delay jitter to be corrected for, which will be necessary as shorter wavelength sources are employed.

\section{Acknowledgements}

The authors would like to acknowledge the help of D. Geissler, A. Staudte, K.T. Kim, T. J. Hammond, A. Avery and D. Crane.

\section{Disclosure statement}

No potential conflict of interest was reported by the authors.

\section{Funding}

This work was supported by NSERC and AFOSR contract [FA9550-13-1-0010].

\section{References}

(1) Griffiths, P.R.; De Haseth, J.A. Fourier transform infrared spectrometry; John Wiley \& Sons: New York, 2007.

(2) Kovačev, M.; Fomichev, S.V.; Priori, E.; Mairesse, Y.; Merdji, H.; Monchicourt, P.; Breger, P.; Norin, J.; Persson, A.; l'Huillier, A.; Wahlstrom, C.G.; Carre, B.; Salieres, P. Phys. Rev. Lett. 2005, 95, 223903.

(3) Witte, S.; Tenner, V.T.; Noom, D.W.; Eikema, K.S. Light Sci. Appl. 2014, 3, e163.

(4) Furukawa, Y.; Nabekawa, Y.; Okino, T.; Saugout, S.; Yamanouchi, K.; Midorikawa, K. Phys. Rev. A 2010, 82, 013421.

(5) Mezger, M.; Jérôme, B.; Kortright, J.B.; Valvidares, M.; Gullikson, E.M.; Giglia, A.; Mahne, N.; Nannarone, S. Phys. Rev. B 2011, 83, 155406.

(6) Spielmann, C.; Burnett, N.; Sartania, S.; Koppitsch, R.; Schnurer, M.; Kan, C.; Lenzner, M.; Wobrauschek, P.; Krausz, F. Science 1997, 278, 661-664.

(7) Nabekawa, Y.; Shimizu, T.; Okino, T.; Furusawa, K.; Hasegawa, H.; Yamanouchi, K.; Midorikawa, K. Phys. Rev. Lett. 2006, 97, 153904.

(8) Nabekawa, Y.; Shimizu, T.; Furukawa, Y.; Takahashi, E.J.; Midorikawa, K. Phys. Rev. Lett. 2009, 102, 213904.

(9) Zerne, R.; Altucci, C.; Bellini, M.; Gaarde, M.B.; Hansch, T.W.; L'Huillier, A.; Lynga, C.; Wahlstrom, C.G. Phys. Rev. Lett. 1997, 79, 1006. 
(10) Descamps, D.; Lyngå, C.; Norin, J.; L'Huillier, A.; Wahlstrom, C.G.; Hergott, J.F.; Merdji, H.; Salieres, P.; Bellini, M.; Hansch, T.W. Opt. Lett. 2000, 25, 135-137.

(11) Lewenstein, M.; Balcou, P.; Ivanov, M.Y.; L'Huillier, A.; Corkum, P.B. Phys. Rev. A 1994, 49, 2117.

(12) Yakovlev, V.S.; Ivanov, M.; Krausz, F. Opt. Express 2007, 15, 15351-15364.

(13) Davis, S.P.; Abrams, M.C.; Brault, J.W. Fourier transform spectrometry, Academic Press: Cambridge, MA, 2001.
(14) Meng, Y.; Zhang, C.; Marceau, C.; Naumov, A.Y.; Corkum, P.B.; Villeneuve, D.M. Opt. Express 2015, 23, 28960-28969.

(15) Kim, K.T.; Zhang, C.; Shiner, A.D.; Kirkwood, S.E.; Frumker, E.; Gariepy, G.; Naumov, A.; Villeneuve, D.M.; Corkum, P.B. Nat. Phys. 2013, 9, 159-163.

(16) Dudovich, N.; Smirnova, O.; Levesque, J.; Mairesse, Y.; Ivanov, M.Y.; Villeneuve, D.M.; Corkum, P.B. Nat. Phys. 2006, 2, 781-786. 